\title{
ISOLATED SINGULARITIES FOR SOLUTIONS OF THE NONLINEAR STATIONARY NAVIER-STOKES EQUATIONS
}

\author{
BY
}

\author{
VICTOR L. SHAPIRO(1)
}

ABSTRACT. The notion for $(u, p)$ to be a distribution solution of the nonlinear stationary Navier-Stokes equations in an open set is defined, and a theorem concerning the removability of isolated singularities for distribution solutions in the punctured open ball $B\left(0, r_{0}\right)-\{0\}$ is established. This result is then applied to the classical situation to obtain a new theorem for the removability of isolated singularities. In particular, in two dimensions this gives a better than expected result when compared with the theory of removable isolated singularities for harmonic functions.

1. Introduction. Let $\Omega$ be a bounded open set in Euclidean $N$-space, $E_{N}$, $N \geq 2$, and let $\mathrm{f}=\left(f_{1}, \ldots, f_{N}\right)$ be a fixed vector in $C^{a}(\Omega), 0<a<1$. (u, p) will be said to be a regular solution of the nonlinear stationary Navier-Stokes equations in $\Omega$ if $u$ is in $\left.C^{2+a} \Omega_{1}\right)$ and $p$ is in $C^{1+a}\left(\Omega_{1}\right)$ for every open subset $\Omega_{1}$ whose closure is contained in $\Omega$ and if, furthermore, $(u, p)$ satisfies

$$
\begin{aligned}
& \nu \Delta u_{j}-u_{k} \partial u_{j} / \partial x_{k}-\partial p / \partial x_{j}+f_{j}=0, \quad j=1, \cdots, N, \\
& \partial u_{k} / \partial x_{k}=0,
\end{aligned}
$$

in $\Omega$, where $\mathbf{u}=\left(u_{1}, \cdots, u_{N}\right), \nu$ is a nonzero constant, and the summation convention is used.

We let $B\left(0, r_{0}\right)$ designate the open $N$-ball with center 0 and radius $r_{0}$ and obtain as a corollary to Theorems 2 and 3 the following result for regular solutions of $(1.1)$ in $B\left(0, r_{0}\right)-\{0\}$.

Received by the editors November 29, 1972 and, in revised form, March 7, 1973.

AMS (MOS) subject classifications (1970). Primary 76D05, 35Q10; Secondary 35D10.

Key words and phrases. Stationary Navier-Stokes equations, isolated singularities, nonlinear, distribution solution.

(1) This research was in part sponsored by the Air Force Office of Scientific Research, Office of Aerospace Research, USAF, under Grant No. AFOSR 71-2046. The United States Government is authorized to reproduce and distribute reprints for Governmental purposes notwithstanding any copyright notation hereon. 
Theorem 1. Let $(\mathrm{u}, p)$ be a regular solution of $(1.1)$ in $B\left(0, r_{0}\right)-\{0\}$ where $f$ is in $C^{a}\left[B\left(0, r_{0}\right)\right], 0<a<1$. Suppose that

(i) there is a $\beta>N$ such that $\mathbf{u}$ is in $L^{\beta}\left[B\left(0, r_{0}\right)\right]$;

(ii) $|\mathbf{u}|=o\left(|x|^{-(N-1) / 2}\right)$ as $|x| \rightarrow 0$ for the case $N=2$;

(iii) $|p|=o\left(|x|^{-(N-1)}\right)$ as $|x| \rightarrow 0$.

Then $(\mathrm{u}, p)$ can be defined at 0 so that $(\mathrm{u}, p)$ is a regular solution of (1.1) in $B\left(0, r_{0}\right)$.

For $N \geq 3$, (ii) is superfluous.

We observe that for $N=3$, Theorem 1 gives an improvement of the result mentioned in the next to the last paragraph of [10]. Also for $N=2$, Theorem 1 gives a better than expected result when compared with the theory of removable isolated singularities for harmonic functions. (See the first paragraph of [10].)

If $(u, p)$ is a regular solution of $(1.1)$ in $\Omega$, then it satisfies

$$
\begin{aligned}
& \nu \Delta u_{j}-\partial\left[u_{j} u_{k}\right] / \partial x_{k}-\partial p / \partial x_{j}+f_{i}=0, \quad j=1, \ldots, N \\
& \partial u_{k} / \partial x_{k}=0
\end{aligned}
$$

in $\Omega$.

In view of this fact, we shall say that $(u, p)$ is a distribution solution of the nonlinear stationary Navier-Stokes equations (1.1) in $\Omega$ [where we now only suppose that $f$ is in $\left.L^{1}(\Omega)\right]$ if $u$ is in $L^{2}(\Omega), p$ is in $L^{1}(\Omega)$, and

$$
\begin{aligned}
& \int_{\Omega}\left[\nu u_{j} \Delta \phi+u_{j} u_{k} \partial \phi / \partial x_{k}+p \partial \phi / \partial x_{j}+\phi f_{j}\right] d x=0, \quad j=1, \ldots, N, \\
& \int_{\Omega}\left[u_{k} \partial \phi / \partial x_{k}\right] d x=0
\end{aligned}
$$

for all $\phi$ in $C_{0}^{\infty}(\Omega)$.

We intend to establish the following result for isolated singularities of distribution solutions of (1.1).

Theorem 2. Let $\mathrm{u}$ be $L^{2}\left[B\left(0, r_{0}\right)\right]$ and $\mathrm{f}$ and $p$ be in $L^{1}\left[B\left(0, r_{0}\right)\right]$. Suppose $(u, p)$ is a distribution solution of the nonlinear stationary Navier-Stokes equations (1.1) in $B\left(0, r_{0}\right)-\{0\}$. Suppose furthermore that

(i) $r^{-N} \int_{B(0, r)}|p| d x=o\left(r^{-(N-1)}\right)$ as $r \rightarrow 0$, and that

(ii) $\left\{r^{-N} \int_{B(0, r)}|u|^{\gamma} d x\right\}^{1 / \gamma}=o\left(r^{-(N-1) / 2}\right)$ as $r \rightarrow 0$ where $\gamma=2$ for $N$ $\geq 3$ and $y>2$ for $N=2$.

Then $(u, p)$ is a distribution solution of (1.1) in $B\left(0, r_{0}\right)$.

We shall also establish the following regularity result for distribution solutions of (1.1).

Theorem 3. Let $\Omega$ be a bounded open set in $E_{N}, N \geq 2$, and assume that $\mathbf{u}$ is in $L^{\beta}(\Omega), \beta>N$, and that $p$ is in $L^{1}(\Omega)$. Suppose furthermore that $(u, p)$ is a distribution solution of the nonlinear stationary Navier-Stokes equations 
(1.1) in $\Omega$, where $\mathrm{f}$ is in $C^{a}(\Omega), 0<a<1$. Then $(\mathrm{u}, p)$ is equal almost every. where in $\Omega$ to a regular solution of (1.1).

It is clear that Theorem 1 is an immediate corollary to Theorems 2 and 3. We establish Theorems 2 and 3 below in $\S 4$ and $\$ 3$, respectively.

2. Fundamental lemmas. In order to establish Theorems 2 and 3 we shall need some results in multiple trigonometric series.

We shall use the following notation: $B(x, r)$ will designate the open $N$-ball with center $x$ and radius $r ; T_{N}$ will designate the $N$-dimensional torus $\{x:-\pi<$ $\left.x_{j} \leq \pi, j=1, \cdots, N\right\} ;(x, y)$ will designate the usual inner product $x_{1} y_{1}+\cdots+$ $x_{N} y_{N}$; and $m$ will designate an integral lattice point.

For $q$ in $L^{1}\left(T_{N}\right)$, we shall set

$$
q^{\hat{q}(m)}=(2 \pi)^{-N} \int_{T_{N}} q(x) e^{-i(m, x)} d x
$$

and designate the Abel partial means of $q$ for $t>0$ by

$$
q(x, t)=\sum_{m} q^{\wedge}(m) e^{i(m, x)-|m| t} .
$$

(Throughout the rest of this paper, we shall not use the summation convention.)

It follows from $[5, \mathrm{p} .56,(17)]$ that

$$
q(x, t)=(2 \pi)^{-N} \int_{T_{N}} q(y) A(x-y, t) d y
$$

where

$$
A(x, t)=\lim _{R \rightarrow \infty} b_{N} t \sum_{|m| \leq R}\left[t^{2}+|x+2 \pi m|^{2}\right]^{-(N+1) / 2}
$$

and $b_{N}$ is a positive constant.

Observing that for $t>0, A(x, t)>0$ for $x$ in $T_{N}$ and that $(2 \pi)^{-N} \int_{T_{N}} A(x, t) d x=1$, we see from (2.3) and (2.4) that

$$
\begin{aligned}
& \text { if } q \text { in } L^{\gamma}\left(T_{N}\right), 1 \leq \gamma<\infty, \text { then } \\
& \lim _{t \rightarrow 0} \int_{T_{N}}|q(x, t)-q(x)|^{\gamma} d x=0 .
\end{aligned}
$$
follows:

Next, for $j=1, \cdots, N$, we introduce the functions $H_{j}(x)$ defined in $E_{N}$ as

$$
H_{j}(x)=\lim _{t \rightarrow 0} \sum_{m \neq 0} i m_{j} e^{i(m, x)-|m| t /|m|^{2}}
$$


From [5, p. 72], we obtain that the following properties prevail:

$$
\begin{aligned}
& H_{j}(x) \text { is in } L^{1}\left(T_{N}\right), \text { and } \\
& H_{j}^{\Upsilon}(m)=i m_{j} /|m|^{2} \text { for } m \neq 0, \\
& H_{j}^{\gamma}(0)=0 .
\end{aligned}
$$

In a similar manner, we introduce the function $H(x)$ defined in $E_{N}$ as follows:

$$
H(x)=\lim _{t \rightarrow 0} \sum_{m \neq 0} e^{i(m, x)-|m| t /|m|^{2} .}
$$

From [5, p. 72], we also obtain that the following properties prevail:

$$
\begin{aligned}
& H(x) \text { is in } L^{1}\left(T_{N}\right), \text { and } \\
& H^{\Upsilon}(m)=|m|^{-2} \text { for } m \neq 0, \\
& H^{\Upsilon}(0)=0 .
\end{aligned}
$$

Also from [5, p. 72], we obtain

$$
\begin{aligned}
& H(x)-|x|^{2} / 2 N \text { and } H_{j}(x) \text { for } j=1, \cdots, N \\
& \text { are harmonic in } E_{N}-\bigcup_{m}\{2 \pi m\},
\end{aligned}
$$

and from (2.6, (2.7), (2.8), (2.9), (2.10), and [6, Lemma 2] that

$$
\partial H(x) / \partial x_{j}=H_{j}(x) \text { for } x \text { in } E_{n}-\bigcup_{m}\{2 \pi m\}
$$

From $\left[5\right.$, p. 72] we also have that there are finite constants $b_{N}^{\prime}, b_{N}^{\prime \prime}, c_{N}^{\prime}$, and $c_{N}^{\prime \prime}$ such that

$$
\begin{array}{r}
\left.\left|H(x)-b_{N}^{\prime}\right| x\right|^{-(N-2)} \mid<c_{N}^{\prime} \text { for } N \geq 3 \\
\left.\left|H(x)-b_{N}^{\prime} \log \right| x\right|^{-1} \mid<c_{N}^{\prime} \text { for } N=2 \\
\left.\left|H_{j}(x)-b_{N}^{\prime} x_{j}\right| x\right|^{-N} \mid<c_{N}^{\prime \prime} \text { for } N \geq 2 \\
\text { for } j=1, \ldots, N \text { and } x \text { in } T_{N}-\{0\} .
\end{array}
$$

Next, using (2.6) through (2.12), we see that the following lemma holds:

Lemma 1. Let $q(x)$ be in $L^{1}\left(T_{N}\right)$, let $0<r_{1}<1$, and let $n$ be a nonnegative integer. Suppose furthermore $q(x)$ is in $C^{n+a}\left[B\left(0, r_{1}\right)\right], 0<a<1$. Set

$$
Q(x)=(2 \pi)^{-N} \int_{T_{N}} q(y) H(x-y) d y
$$

and 


$$
Q_{j}(x)=(2 \pi)^{-N} \int_{T_{N}} q(y) H_{j}(x-y) d y \quad \text { for } j=1, \ldots, N .
$$

Then $Q(x)$ is in $C^{n+2+a}[B(0, r)]$ and $Q_{j}(x)$ is in $C^{n+1+a}[B(0, r)]$ for every $r$ such that $0<r<r_{1}$.

Next, we intend to establish the following lemma:

Lemma 2. Let $q(x)$ be in $L^{1}\left(T_{N}\right)$ and let $0<r_{1}<1$. Suppose that $q(x)$ is in $L^{\gamma}\left[B\left(0, r_{1}\right)\right]$ where $1<y<N$. For $j=1, \cdots, N$, set

$$
Q_{j}(x)=(2 \pi)^{-N} \int_{T_{N}} q(y) H_{j}(x-y) d y .
$$

Then $Q_{j}(x)$ is in $L^{N \gamma /(N-\gamma)}[B(0, r)]$ for every $r$ such that $0<r<r_{1}$

Let

$$
0<r_{4}<r_{3}<r_{2}<r_{1}
$$

The lemma will be established if we can show

$$
Q_{j}(x) \text { is in } L^{N \gamma /(N-\gamma)}\left[B\left(0, r_{4}\right)\right]
$$

In order to do this, we choose a function $\lambda(x)$ such that

$$
\begin{aligned}
\lambda(x) & =1 \text { for } 0 \leq|x| \leq r_{3} \\
& =0 \text { for } r_{2} \leq|x|<\infty \text { and } \\
\lambda(x) & \text { in } C^{\infty}\left(E_{N}\right) .
\end{aligned}
$$

Next, we set

$$
\begin{aligned}
& Q_{j}^{\prime}(x)=(2 \pi)^{-N} \int_{T_{N}} \lambda(y) q(y) H_{j}(x-y) d y \text { and } \\
& Q_{j}^{\prime \prime}(x)=(2 \pi)^{-N} \int_{T_{N}}[1-\lambda(y)] q(y) H_{j}(x-y) d y .
\end{aligned}
$$

From (2.12), we have there is a constant $c_{N}$ such that

$$
\left|H_{j}(y)\right| \leq c_{N}|y|^{-(N-1)} \text { for } y \text { in } T_{N}-\{0\} .
$$

From (2.16), (2.17), and (2.18), we have that

$$
\left|Q_{j}^{\prime}(x)\right| \leq c_{N}(2 \pi)^{-N} \int_{B\left(0, r_{2}\right)}|\lambda(y) q(y)||x-y|^{-(N-1)} d y
$$


Consequently, it follows from [3, Lemma 5, p. 13] and the hypothesis of the lemma that

$$
Q_{j}^{\prime} \text { is in } L^{N \gamma /(N-\gamma)}\left[B\left(0, r_{4}\right)\right] \text {. }
$$

Also from (2.10), (2.16), and (2.17), we have that

$$
Q_{j}^{\prime \prime} \text { is in } C^{\infty}\left[B\left(0, r_{3}\right)\right]
$$

From (2.13) and (2.17), we have that $Q_{j}=Q_{j}^{\prime}+Q_{j}^{\prime \prime}$ whenever both $Q_{j}^{\prime}$ and $Q_{j}^{n}$ are finite. But then (2.15) follows immediately from (2.20) and (2.21), and the proof to the lemma is complete.

Lemma 3. Let $q, r_{1}$, and $Q_{j}$ be as in Lemma 2 except now suppose that $N<\gamma<\infty$. Then $Q_{j}$ is in $C^{\delta}[B(0, r)]$ for every $\delta$ such that $0<\delta<1-(N / \gamma)$ and for every $r$ such that $0<r<r_{1}$.

Once again we suppose that $(2.14)$ holds. Then the le mma will be established if we can show

$$
Q_{j} \text { is in } C^{\delta}\left[B\left(0, r_{4}\right)\right] \text { for } 0<\delta<1-(N / \gamma) \text {. }
$$

With $b_{N}^{\prime \prime}$ as in (2.12), set

$$
H_{j 1}(x)=H_{j}(x)-b_{N}^{\prime \prime} x_{j}|x|^{-N} \text { for } x \text { in } B(0,2)-\{0\} .
$$

Then it follows from (2.10) and (2.12) that $H_{j 1}$ can be defined at 0 so that

$$
H_{j 1} \text { is harmonic in } B(0,2) \text {. }
$$

Next, with $\lambda$ defined as in (2.16), we set for $x$ in $B\left(0, r_{2}\right)$

$$
Q_{j 1}(x)=(2 \pi)^{-N} \int_{B\left(0, r_{2}\right)} \lambda(y)_{q}(y) H_{j 1}(x-y) d y
$$

and

$$
Q_{j 2}(x)=(2 \pi)^{-N} \int_{B\left(0, r_{2}\right)} \lambda(y) q(y)\left(x_{j}-y_{j}\right)|x-y|^{-N} d y \text {. }
$$

With $Q_{j}^{\prime \prime}$ defined as in (2.17), we see from (2.13), (2.16), (2.25), and (2.26) that

$$
Q_{j}(x)=Q_{j 1}(x)+Q_{j 2}(x)+Q_{j}^{\prime \prime}(x) \text { for } x \text { in } B\left(0, r_{3}\right)
$$


Now as before (2.21) holds. From (2.24) and (2.25) we see that

$$
Q_{j 1} \text { is harmonic in } B\left(0, r_{3}\right) \text {. }
$$

On the other hand, from (2.16), (2.26), the hypothesis of the lemma [3, Lemma 5, p. 13] and the follow ing inequality (see $[8,(21)$, p. 221])

$$
\left|\left(x_{j}+b_{j}\right)\right| x+\left.b\right|^{-N}-\left.x_{j}|x|^{-N}|\leq K| b\right|^{\delta}\left[|x+b|^{-(N-1+\delta)}+|x|^{-(N-1+\delta)}\right]
$$

where $K$ is a constant independent of $x$ and $b$, we see that

$$
Q_{j 2} \text { is in } C^{\delta}\left[B\left(0, r_{4}\right)\right] \text { for } 0<\delta<1-(N / \gamma) \text {. }
$$

But then (2.22) follows from (2.21), (2.27), (2.28) and (2.29), and the proof of the lemma is complete.

Next, we set

$$
K_{j k}(x)=x_{j} x_{k} /|x|^{2+N} \text { for } x \neq 0 \text { and } j, k=1, \cdots, N, j \neq k,
$$

and observe that $K_{j k}(x)$ is a spherical harmonic Calderón-Zygmund kernel (see $[1$, p. 261]). We then define for $j \neq k$ and for $x \neq 2 \pi m$

$$
\begin{aligned}
K_{j k}^{* *}(x) & =\lim _{R \rightarrow \infty} \sum_{1 \leq|m| \leq R}\left[K_{j k}(x+2 \pi m)-K_{j k}(2 \pi m)\right] \text { and } \\
K_{j k}^{*}(x) & =K_{j k}(x)+K_{j k}^{* *}(x) .
\end{aligned}
$$

Also, we observe from $[1$, p. 252$]$ that the series defining $K_{j k}^{* *}(x)$ is absolutely convergent for $x$ in $T_{N}^{2}$ where

(2.31') $\quad T_{N}^{2}=\left\{z: z=x+y,|x|<2\right.$ and $\left|y_{j}\right|<\pi$ for $\left.j=1, \cdots, N\right\}$.

In particular, we note that $K_{j}^{* *}(0)$ is defined.

Next, we observe from [1, pp. 257-261] that

$$
\begin{array}{rlr}
\lim _{\epsilon \rightarrow 0}(2 \pi)^{-N} & \int_{T_{N}-B(0, \epsilon)} K_{j k}^{*}(x) e^{i(m, x)} d x \\
& =\xi_{j k} m_{j} m_{k}|m|^{-2} & \text { for } m \neq 0 \\
& =0 & \text { for } m=0
\end{array}
$$

where $\xi_{j k}$ is a nonzero constant.

We next establish the following lemma: 
Lemma 4. Let $q(x)$ be in $L^{1}\left(T_{N}\right)$ and let $0<r_{1}<1$. Suppose that $q(x)$ is in $L^{\gamma}\left[B\left(0, r_{1}\right)\right]$ where $1<\gamma<\infty$. Then for almost every $x$ in $B\left(0, r_{1}\right)$ the following limit exists and is finite:

$$
q_{j k}(x)=\lim _{\epsilon \rightarrow 0} \xi_{j k}^{-1}(2 \pi)^{-N} \int_{T_{N}-B(x, \epsilon)} q(y) K_{j k}^{*}(x-y) d y .
$$

Furtbermore, $q_{j k}$ is in $L^{\gamma}[B(0, r)]$ for every $r$ such that $0<r<r_{1}$.

Once again we suppose that (2.14) holds. The lemma will be established if we show that

(2.34) the limit in (2.33) is finite valued almost everywhere in $B\left(0, r_{4}\right)$

and

$$
q_{j k} \text { is in } L^{\gamma}\left[B\left(0, r_{4}\right)\right]
$$

We let $\lambda(x)$ be as in (2.16) and we set

$$
q_{j k}^{\prime}(x)=\lim _{\epsilon \rightarrow 0} \xi_{j k}^{-1}(2 \pi)^{-N} \int_{T_{N^{-B}}(x, \epsilon)} \lambda(y) q(y) K_{j k}^{*}(x-y) d y .
$$

Since $\lambda(y) q(y)$ is in $L^{\gamma}\left(T_{N}\right)$, we have from [1, Theorem 1, p. 253] that

$$
q_{j k}^{\prime}(x) \text { exists and is finite almost everywhere in } T_{N} \text {, }
$$

and from [1, Theorem 2, p. 253] that

$$
q_{j k}^{\prime}(x) \text { is in } L^{\gamma}\left(T_{N}\right)
$$

Next, we set

$$
q_{j k}^{\prime \prime}(x)=\lim _{\epsilon \rightarrow 0} \xi_{j k}^{-1}(2 \pi)^{-N} \int_{T_{N^{-B(x, \epsilon)}}}[1-\lambda(y)]_{q}(y) K_{j k}^{*}(x-y) d y .
$$

Since for $z \neq 0$ and $z$ in $T_{N}^{2}$ [where $T_{N}^{2}$ is defined in $\left(2.31^{\prime}\right)$ ], we have that $K_{j k}^{*}(z)=K_{j k}(z)+K_{j k}^{* *}(z)$ and since furthermore $K_{j k}^{* *}(z)$ is continuous in $T_{N}^{2}$, we conclude from (2.16) and (2.39) that

$$
q_{j k}^{\prime \prime}(x) \text { is continuous in } B\left(0, r_{3}\right) \text {. }
$$

But then from (2.33), (2.36), and (2.39) we have that $q_{j k}(x)=q_{j k}^{\prime}(x)+q_{j k}^{\prime \prime}(x)$ whenever both $q_{j k}^{\prime}(x)$ and $q_{j k}^{\prime \prime}(x)$ exist and are finite. Consequently (2.34) follows from (2.14), (2.37), and (2.40). Also (2.35) follows from (2.14), (2.38), 
and (2.40), and the proof of the lemma is complete.

Next, we state the following lemma.

Lemma 5. Let $q(x)$ be in $L^{1}\left(T_{N}\right)$ and let $0<r_{1}<1$. Suppose that $q(x)$ is cont inuous in $B\left(0, r_{1}\right)$. For $t>0$ and $j \neq k$ where $j, k=1, \cdots, N$, set

$$
q_{j k}(x, t)=\sum_{m \neq 0} q^{\Upsilon}(m) m_{j} m_{k}|m|^{-2} e^{i(m, x)-|m| t} .
$$

Then

$$
\begin{gathered}
\lim _{t \rightarrow 0}\left[q_{j k}(x, t)-(2 \pi)^{-N} \xi_{j k}^{-1} \int_{T_{N}-B(x, t)} q(y) K_{j k}^{*}(x-y) d y\right] \\
=0 \text { for } x \text { in } B\left(0, r_{1}\right) .
\end{gathered}
$$

The above lemma follows from the material in [4, pp. 44-46]. We leave the details to the reader.

Next, we establish the following lemma.

Lemma 6. Let $q(x)$ be in $L^{1}\left(T_{N}\right)$ and let $0<r_{1}<1$. For $t>0$ and $j \neq k$ where $j, k=1, \cdots, N$, let $q_{j k}(x, t)$ be defined by (2.41). Suppose that $q(x)$ is in $L^{\gamma}\left[B\left(0, r_{1}\right)\right]$ where $1<\gamma<\infty$. Then for almost every $x$ in $B\left(0, r_{1}\right)$,

$$
\lim _{t \rightarrow 0} q_{j k}(x, t)=q_{j k}(x)
$$

where $q_{j k}(x)$ is defined almost everywhere by the limit in (2.33).

Once again we suppose that (2.14) holds. The lemma will be established if we show

$$
\text { for almost every } x \text { in } B\left(0, r_{4}\right), \lim _{t \rightarrow 0} q_{j k}(x, t)=q_{j k}(x) \text {. }
$$

To establish (2.42), we let $\lambda$ be as in (2.16) and set

$$
q_{j k}^{\prime \prime}(x, t)=\sum_{m \neq 0}\left[q^{\Upsilon}(m)-\left(\lambda_{q}\right)^{\wedge}(m)\right] m_{j} m_{k}|m|^{-2} e^{i(m, x)-|m| t}
$$

Since $[1-\lambda(x)] q(x)=0$ in $B\left(0, r_{3}\right)$, it follows from (2.39), (2.40), and Lemma 5 that

$$
\lim _{t \rightarrow 0} q_{j k}^{\prime \prime}(x, t)=q_{j k}^{\prime \prime}(x) \text { for } x \text { in } B\left(0, r_{3}\right)
$$

Next, we set

$$
q_{j k}^{\prime}(x, t)=\sum_{m \neq 0}(\lambda q)^{\wedge}(m) m_{j} m_{k}|m|^{-2} e^{i(m, x)-|m| t}
$$


and obtain from [4, pp. 44-46] (or from [1] and [5, pp. 55-56]) that

$$
\begin{gathered}
\lim _{t \rightarrow 0}\left\{q_{j k}^{\prime}(x, t)-\xi_{j k}^{-1}(2 \pi)^{-N} \int_{T_{N^{-B(x, t)}}} \lambda(y) q(y) K_{j k}^{*}(x-y) d y\right\} \\
=0 \text { almost everywhere in } T_{N} .
\end{gathered}
$$

But then it follows from (2.36), (2.37), and (2.46) that

$$
\lim _{t \rightarrow 0} q_{j k}^{\prime}(x, t)=q_{j k}^{\prime}(x) \text { almost everywhere in } T_{N} \text {. }
$$

From (2.41), (2.43), and (2.45), we have that

$$
q_{j k}(x, t)=q_{j k}^{\prime}(x, t)+q_{j k}^{\prime \prime}(x, t)
$$

On the other hand, from (2.33), (2.36), and (2.39) we have that $q_{j k}(x)=q_{j k}^{\prime}(x)+$ $q_{j k}^{\prime \prime}(x)$ whenever both $q_{j k}^{\prime}(x)$ and $q_{j k}^{\prime \prime}(x)$ exist and are finite. But then (2.42) follows from this fact, $(2.37),(2.40),(2.44)$ and $(2.47)$. The proof of the lemma is consequently complete.

Next, we establish the analogue of Lemma 4 for Hölder continuous functions.

Lemma 7. Let $q(x)$ be in $L^{1}\left(T_{N}\right)$ and let $0<r_{1}<1$. Suppose that $q(x)$ is in $C^{\gamma}\left[B\left(0, r_{1}\right)\right], 0<\gamma<1$. Then the limit in (2.33), called $q_{j k}(x)$, exists and is finite for every $x$ in $B\left(0, r_{1}\right)$. Furthermore $q_{j k}(x)$ is in $C^{\gamma}[B(0, r)]$ for every $r$ such that $0<r<r_{1}$.

Once again we suppose that (2.14) holds. The lemma will be established if we show that the limit in $(2.33)$ is finite valued in $B\left(0, r_{4}\right)$,

and

$$
q_{j k} \text { is in } C^{\gamma}\left[B\left(0, r_{4}\right)\right] \text {. }
$$

We let $\lambda(x)$ be as in (2.16) and we define $q_{j k}^{\prime}(x)$ by the limit in (2.36). From [1, Theorem 11, p. 262] we have

$$
q_{j k}^{\prime}(x) \text { exists and is finite everywhere in } B\left(0, r_{1}\right)
$$

and furthermore

$$
q_{j k}^{\prime}(x) \text { is in } C^{\gamma}\left[B\left(0, r_{1}\right)\right]
$$


Next, for $x$ in $B\left(0, r_{3}\right)$ we define $q_{j k}^{\prime \prime}(x)$ by the limit in (2.39). Since $\lambda(y)=$ 1 in $B\left(0, r_{3}\right)$, we have that

$$
q_{j k}^{\prime \prime}(x) \text { exists and is finite everywhere in } B\left(0, r_{3}\right) .
$$

Furthermore from (2.31), we have that for $x$ in $B\left(0, r_{3}\right)$

$$
\begin{aligned}
& q_{j k}^{\prime \prime}(x)=\xi_{j k}^{-1}(2 \pi)^{-N} \int_{T_{N}-B\left(0, r_{3}\right)}[1-\lambda(y)] q(y) K_{j k}(x-y) d y \\
& +\xi_{j k}^{-1}(2 \pi)^{-N} \int_{T_{N}-B\left(0, r_{3}\right)}[1-\lambda(y)]_{q}(y) K_{j k}^{* *}(x-y) d y .
\end{aligned}
$$

But an easy computation shows that $K_{j k}^{* *}(z)$ is in $C^{1}\left(T_{N}^{2}\right)$ where $T_{N}^{2}$ is defined by $\left(2.31^{\prime}\right)$. Consequently, it follows from (2.54) that

$$
q_{j k}^{\prime \prime}(x) \text { is in } C^{1}\left[B\left(0, r_{4}\right)\right] \text {. }
$$

Since $q_{j k}(x)=q_{j k}^{\prime}(x)+q_{j k}^{\prime \prime}(x)$ whenever both $q_{j k}^{\prime}(x)$ and $q_{j k}^{\prime \prime}(x)$ exist and are finite, (2.49) follows from (2.51) and (2.53). Also (2.50) follows from (2.52) and (2.55). The proof of the lemma is consequently complete.

Next, we establish the analogue of Lemma 6 for Hölder continuous functions.

Lemma 8. Let $q(x)$ be in $L^{1}\left(T_{N}\right)$ and let $0<r_{1}<1$. For $t>0$ and $j \neq k$ where $j, k=1, \cdots, N$, let $q_{j k}(x, t)$ be defined by (2.41). Suppose that $q(x)$ is in $C^{\gamma}\left[B\left(0, r_{1}\right)\right]$ where $0<y<1$. Then for every $x$ in $B\left(0, r_{1}\right)$,

$$
\lim _{t \rightarrow 0} q_{j k}(x, t)=q_{j k}(x)
$$

where $q_{j k}(x)$ is defined everywhere in $B\left(0, r_{1}\right)$ by the limit in (2.33).

To establish the above lemma, we observe from its hypothesis and from Lemma 5 that

$$
\begin{gathered}
\lim _{t \rightarrow 0}\left[q_{j k}(x, t)-\xi_{j k}^{-1}(2 \pi)^{-N} \int_{T_{N}-B(x, t)} q(y) K_{j k}^{*}(x-y) d y\right] \\
=0 \text { everywhere in } B\left(0, r_{1}\right) .
\end{gathered}
$$

Invoking Lemma 7, we conclude from this last fact that

$$
\lim _{t \rightarrow 0}\left[q_{j k}(x, t)-q_{j k}(x)\right]=0 \text { everywhere in } B\left(0, r_{1}\right) .
$$

This completes the proof of the lemma.

Next, we set 


$$
K_{j}(x)=\left.\left\{(N-1) x_{j}^{2}-\sum_{k=1 ; j \neq k}^{N} x_{k}^{2}\right\}|| x\right|^{2+N} \text { for } x \neq 0, j=1, \cdots, N .
$$

We then define, for $x \neq 2 \pi m$,

$$
\begin{aligned}
& K_{j}^{* *}(x)=\lim _{R \rightarrow \infty} \sum_{1 \leq|m| \leq R}\left[K_{j}(x+2 \pi m)-K_{j}(2 \pi m)\right] \text { and } \\
& K_{j}^{*}(x)=K_{j}(x)+K_{j}^{* *}(x)
\end{aligned}
$$

and observe as before that the series defining $K_{j}^{* *}(x)$ is absolutely convergent for $x$ in $T_{N}^{2}$, and in particular that $K_{j}^{* *}(0)$ is defined.

Now $K_{j}(x)$ is a spherical harmonic Calderón-Zygmund kernel (see [1, p. 261]). As a consequence we have from $[1, \mathrm{pp} .257-261]$ that

$$
\begin{array}{ll}
\lim _{\epsilon \rightarrow 0}(2 \pi)^{-N} \int_{T_{N}-B(0, \epsilon)} K_{j}^{*}(x) e^{-i(m, x)} d x & \\
\quad=\xi_{j}\left[(N-1) m_{j}^{2}-\sum_{k=1 ; a \neq k}^{N} m_{k}^{2}\right]|m|^{-2} & \text { for } m \neq 0, \\
\quad=0 & \text { for } m=0,
\end{array}
$$

where $\xi_{j}$ is a nonzero constant.

The following two lemmas follow in a manner similar to that in which Lemmas $4,6,7$, and 8 were established. We leave the details to the reader.

Lemma 9. Let $q$ be in $L^{1}\left(T_{N}\right)$ and let $0<r_{1}<1$. Suppose that $q(x)$ is in $L^{\gamma}\left[B\left(0, r_{1}\right)\right]$ where $1<\gamma<\infty$. Then for almost every $x$ in $B\left(0, r_{1}\right)$ the following limit exists and is finite.

$$
q_{j}(x)=\lim _{\epsilon \rightarrow 0} \xi_{j}^{-1}(2 \pi)^{-N} \int_{T_{N}-B(x, \epsilon)} q(y) K_{j}^{*}(x-y) d y .
$$

Also, $q_{j}$ is in $L^{\gamma}[B(0, r)]$ for every $r$ sucb that $0<r\left\langle r_{1}\right.$. Furthermore for $t>$ 0 , set

$$
q_{j}(x, t)=\sum_{m \neq 0} q^{\wedge}(m)\left[(N-1) m_{j}^{2}-\sum_{k=1, j \neq k}^{N} m_{k}^{2}\right]|m|^{-2} e^{i(m, x)-|m| t} .
$$

Then for almost every $x$ in $B\left(0, r_{1}\right), \lim _{t \rightarrow 0} q_{j}(x, t)=q_{j}(x)$.

Lemma 10. Let $q(x)$ be in $L^{1}\left(T_{N}\right)$ and let $0<r_{1}<1$. Suppose that $q(x)$ is in $C^{\gamma}\left[B\left(0, r_{1}\right)\right], 0<\gamma<1$. Then the limit in (2.59), called $q_{j}(x)$, exists and is finite for every $x$ in $B\left(0, r_{1}\right)$. Also, $q_{j}(x)$ is in $C^{\gamma}[B(0, r)]$ for every $r$ sucb that $0<r<r_{1}$. Furthermore with $q_{j}(x, t)$ defined by $(2.60), \lim _{t \rightarrow 0} q_{j}(x, t)=$ $q_{j}(x)$ for every $x$ in $B\left(0, r_{1}\right)$. 
Next, we establish the following lemma.

Lemma 11. Let $q(x)$ be in $L^{1}\left(T_{N}\right)$ and let $0<r_{1}<1$. For $j=1, \cdots, N$, and $t>0$, set

$$
q_{j j}(x, t)=\sum_{m \neq 0} q^{\wedge}(m) m_{j}^{2}|m|^{-2} e^{i(m, x)-|m| t} .
$$

(a) Suppose $q$ is in $L^{\gamma}\left[B\left(0, r_{1}\right)\right], 1<\gamma<\infty$. Then there exists a function $q_{j j}(x)$ in $L^{\gamma}[B(0, r)]$ for every $r$ such that $0<r<r_{1}$ with the property that $\lim _{t \rightarrow 0} q_{j j}(x, t)=q_{j j}(x)$ almost everywhere in $B\left(0, r_{1}\right)$. Furthermore, $q_{j j}(x)=$ $\left[q(x)-q^{\prime}(0)+q_{j}(x)\right] / N$ almost everywhere in $B\left(0, r_{1}\right)$ where $q_{j}(x)$ is defined by the limit in (2.59).

(b) Suppose $q$ is in $C^{\gamma}\left[B\left(0, r_{1}\right)\right], 0<y<1$. Then there exists a function $q_{j j}(x)$ in $C^{\gamma}[B(0, r)]$ for every $r$ such that $0<r<r_{1}$ with the property that $\lim _{t \rightarrow 0} q_{j j}(x, t)=q_{j j}(x)$ everywhere in $B\left(0, r_{1}\right)$. Furthermore, $q_{j j}(x)=$ $\left[q(x)-q^{\alpha}(0)+q_{j}(x)\right] / N$ everywhere in $B\left(0, r_{1}\right)$.

We first establish (a) of Lemma 11. Since this part of the lemma is true for the special case $q \equiv$ constant in $T_{N}$, we see from the start that with no loss in generality, we can assume that

$$
q^{\wedge}(0)=0
$$

Next, we observe that

$$
m_{j}^{2}=\left\{|m|^{2}+\left[(N-1) m_{j}^{2}-\sum_{k=1 ; j \neq k}^{N} m_{k}\right]\right\} / N .
$$

We consequently conclude from (2.2), (2.60), (2.61), (2.62), and (2.63) that

$$
q_{j j}(x, t)=\left[q(x, t)+q_{j}(x, t)\right] / N .
$$

From [5, The orem 2, pp. 55-56], we have that

$$
\lim _{t \rightarrow 0} q(x, t)=q(x) \quad \text { almost everywhere in } B\left(0, r_{1}\right) .
$$

From Lemma 9, we have that

$$
\lim _{t \rightarrow 0} q_{j}(x, t)=q_{j}(x) \quad \text { almost everywhere in } B\left(0, r_{1}\right) \text {. }
$$

We conclude from (2.64), (2.65), and (2.66) that 


$$
\lim _{t \rightarrow 0} q_{j j}(x, t)=q(x)+q_{j}(x) \text { almost everywhere in } B\left(0, r_{1}\right) .
$$

The conclusion to (a) of Lemma 11 then follows immediately from the hypothesis, (2.62), (2.67), and Lemma 9.

The proof of (b) of Lemma 11 follows in a similar manner. In (2.65) and (2.66), we replace almost everywhere by everywhere, and we use Lemma 10 instead of Lemma 9. We leave the details to the reader.

Next, we state the following lemma.

Lemma 12. Let $q(x)$ be in $L^{1}\left(T_{N}\right)$ and let $0<r_{1}<1$. For $j, k=1, \cdots, N$ and $t>0$, set

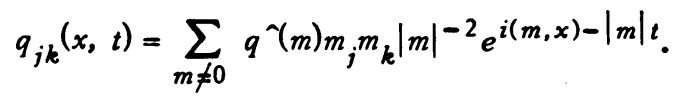

Suppose that $q(x)$ is in $L^{\gamma}\left[B\left(0, r_{1}\right)\right], 1<\gamma<N /(N-1)$, and that

$$
\left\{r^{-N} \int_{B(0, r)}|q(x)|^{\gamma} d x\right\}^{1 / \gamma}=o\left(r^{-(N-1)}\right) \text { as } r \rightarrow 0 \text {. }
$$

Then there exists a function $q_{j k}(x)$ which is in $L^{\gamma}[B(0, r)]$ for every $r$ sucb that $0<r<r_{1}$ with the property that $\lim _{t \rightarrow 0} q_{j k}(x, t)=q_{j k}(x)$ almost everywbere in $B\left(0, r_{1}\right)$. Furthermore

$$
\left\{r^{-N} \int_{B(0, r)}\left|q_{j k}(x)\right|^{\gamma} d x\right\}^{1 / \gamma}=o\left(r^{-(N-1)}\right) \text { as } r \rightarrow 0
$$

Lemma 12 follows from the techniques used to establish Lemmas 3, 4, and 11 (a) and [9, Lemma 5.1, p. 206]. We leave the details to the reader.

Next, we state the following lemma.

Lemma 13. Let $q(x)$ be in $L^{1}\left(T_{N}\right)$ and define $Q(x)$ and $Q_{j}(x)$ for $j=1$, $\cdots, N$ almost everywhere in $T_{N}$ by setting

$$
Q_{j}(x)=(2 \pi)^{-N} \int_{T_{N}} q(y) H_{j}(x-y) d y
$$

and

$$
Q(x)=(2 \pi)^{-N} \int_{T_{N}} q(y) H(x-y) d y
$$

Then $Q(x)$ and $Q_{j}(x)$ are in $L^{1}\left(T_{N}\right)$ and as $r \rightarrow 0$,

$$
r^{-N} \int_{B(0, r)}\left|Q_{j}(x)\right| d x=o\left(r^{-(N-1)}\right)
$$


and

$$
\begin{aligned}
r^{-N} \int_{B(0, r)}|Q(x)| d x & =o\left(r^{-(N-2)}\right) \text { for } N \geq 3 \\
& =o\left(\log r^{-1}\right) \text { for } N=2 .
\end{aligned}
$$

The proof of $(2.68)$ is very similar to that given on $[7$, p. 90]. (2.69) follows in an a nalogous manner.

3. Proof of Theorem 3. To prove Theorem 3, it is clear that we can assume with no loss in generality that $\Omega=B\left(0, r_{0}\right)$ where $0<r_{0}<1$. We let $0<r_{1}<r_{0}$ and observe that the proof will be complete if we can show that there is a vector field $u^{\prime}$ and a function $p^{\prime}$ such that $u^{\prime}$ is in $C^{2+\alpha}[B(0, r)]$ and $p^{\prime}$ is in $C^{1+a}[B(0, r)]$ for every $r$ such that $0<r<r_{1}$, suth that $\left(u^{\prime}, p^{\prime}\right)$ satisfy $(1.1)$ in $B\left(0, r_{1}\right)$ and such that $u^{\prime}=u$ and $p^{\prime}=p$ almost everywhere in $B\left(0, r_{1}\right)$. In order to accomplish this fact, we introduce

$$
r_{1}<r_{1}^{\prime}<r_{0}^{\prime}<r_{0}<1
$$

and choose a function $\eta(x)$ which is in $C^{\infty}\left(E_{N}\right)$, which equals one in $B\left(0, r_{1}^{\prime}\right)$ and equals zero in $E_{N}-B\left(0, r_{0}^{\prime}\right)$. We then define a periodic vector field $u^{*}(x)$ as follows:

$$
\begin{aligned}
\mathbf{u}^{*}(x) & =\eta(x) \mathbf{u}(x) & & \text { for } x \text { in } B\left(0, r_{0}\right), \\
& =0 & & \text { for } x \text { in } T_{N}-B\left(0, r_{0}\right), \\
\mathbf{u}^{*}(x) & =\mathbf{u}^{*}(x-2 \pi m) & & \text { for } x \text { in } T_{N}+2 \pi m .
\end{aligned}
$$

We define $p^{*}(x)$ and $f^{*}(x)$ in a similar manner and observe that $\left(u^{*}, p^{*}\right)$ is a distribution solution of $(1.1)$ in $B\left(0, r_{1}\right)$ with $f$ replaced by $f^{*}$, i.e. $\left(u^{*}, p^{*}\right)$ satisfies (1.3) with $\Omega=B\left(0, r_{1}\right)$ and $f$ replaced by $f^{*}$.

It then follows that Theorem 3 will be established if we establish the following lemma.

Lemm a 14. Let $\mathrm{u}, p$ and $\mathrm{f}$ be defined in $E_{N}$ and be periodic of period $2 \pi$ in each variable. Assume also that $u$ is in $L^{\beta}\left(T_{N}\right), \beta>N$, that $p$ is in $L^{1}\left(T_{N}\right)$ and that $\mathrm{f}$ is in $C^{a}\left(E_{N}\right), 0<\alpha<1$. Suppose that $(u, p)$ is a distribution solution of (1.1) in $B\left(0, r_{1}\right), 0<r_{1}<1$, i.e., (u,p) satisfies (1.3) with $\Omega=B\left(0, r_{1}\right)$. Then $(u, p)$ is almost everywhere equal to a regular solution of $(1.1)$ in $B\left(0, r_{1}\right)$.

We see that in order to establish Lemma 14, we can assume from the start with no loss in generality that

$$
\hat{f_{j}}(0)=0, \quad j=1, \cdots, N .
$$


We first intend to establish

$$
p(x) \text { is in } L^{\beta / 2}[B(0, r)] \text { for every } r \text { such that } 0<r<r_{1} \text {. }
$$

For ease of notation for $0<r<1$, we shall say

$$
\begin{aligned}
& \phi \text { is in } C_{0}^{\infty}\left[r, T_{N}\right] \text { if } \phi \text { is in } C_{0}^{\infty}[B(0, r)] \text { and } \\
& \phi=0 \text { in } T_{N}-B(0, r) .
\end{aligned}
$$

We next observe from (2.5) that if $q$ is in $L^{1}\left(T_{N}\right), \phi$ is in $C_{0}^{\infty}\left[r_{1}, T_{N}\right]$, and $q(x, t)$ is given by (2.2) that

$$
\int_{T_{N}} q(x) \frac{\partial \phi(x)}{\partial x j} d x=\lim _{t \rightarrow 0} \int_{T_{N}} q(x, t) \frac{\partial \phi(x)}{\partial x j} d x
$$

$$
=-(2 \pi)^{N} \sum_{m} q^{\wedge}(m) i m_{j} \phi^{\wedge}(-m) .
$$

As a consequence, we conclude from the hypothesis of Lemma 14, from (1.3), and from (3.1) that, for $\phi$ in $C_{0}^{\infty}\left[r_{1}, T_{N}\right]$,

$$
\begin{array}{r}
\sum_{m \neq 0}\left\{\nu u_{j}^{\wedge}(m)|m|^{2}+i \sum_{k=1}^{N}\left(u_{j} u_{k}\right)^{\wedge}(m)_{m_{k}}+i m_{j} p^{\wedge}(m)-\hat{f_{j}(m)}\right\} \\
\\
\text { for } j=1, \ldots, N,
\end{array}
$$

and

$$
\sum_{m \neq 0} i\left(\sum_{j=1}^{N} m_{j} u_{j}^{\curlyvee}(m)\right) \phi^{\Upsilon}(-m)=0 .
$$

(We shall not use the summation convention in establishing Lemma 14.) Next, with $H_{j}(x)$ defined by $(2.6)$, we set

$$
P_{j}(x)=(2 \pi)^{-N} \int_{T_{N}} p(y) H_{j}(x-y) d y
$$

and

$$
U_{j k}(x)=(2 \pi)^{-N} \int_{T_{N}} u_{j}(y) u_{k}(y) H_{k}(x-y) d y .
$$

Wi th $H(x)$ defined by $(2.8)$, we set

$$
\mathcal{F}_{j}(x)=(2 \pi)^{-N} \int_{T_{N}} f_{j}(y) H(x-y) d y
$$

and then we define 


$$
v_{j}(x)=P_{j}(x)+\sum_{k=1}^{N} U_{j k}(x)-\mathcal{F}_{j}(x) .
$$

Now it follows from (3.7), 3.8), (3.9), (3.10) that

$$
\begin{aligned}
v_{j}^{\Upsilon}(m) & =\left[i m_{j} p^{\wedge}(m)+i \sum_{k=1}^{N} m_{k}\left(u_{j} u_{k}\right)^{\wedge}(m)-\hat{f_{j}^{\wedge}(m)}\right]|m|^{-2} \text { for } m \neq 0, \\
& =0 \text { for } m=0 .
\end{aligned}
$$

As a consequence, we obtain from (3.5) and (3.11) that

$$
\sum_{m \neq 0}\left[\nu u_{j}^{\curlyvee}(m)+v_{j}^{\wedge}(m)\right]|m|^{2} \phi^{\curlyvee}(-m)=0 \quad \text { for } j=1, \cdots, N \text {. }
$$

Now (3.12) implies that

$$
\int_{B\left(0, r_{1}\right)}\left[\nu u_{j}(x)+v_{j}(x)\right] \Delta \phi(x) d x=0, \quad j=1, \ldots, N,
$$

for all $\phi$ in $C_{0}^{\infty}\left[r_{1}, T_{N}\right]$.

We conclude from Weyl's lemma $[2$, p. 199] that for $j=1, \cdots, N$ there is a function $w_{j}(x)$ such that

$$
\begin{aligned}
& w_{j}(x) \text { is harmonic in } B\left(0, r_{1}\right) \text { and equal } \\
& \text { almost everywhere in } T_{N} \text { to } \nu u_{j}(x)+v_{j}(x) .
\end{aligned}
$$

Next, we set

$$
\mathcal{U}(x)=\sum_{j=1}^{N}(2 \pi)^{-N} \int_{T_{N}} u_{j}(y) H_{j}(x-y) d y
$$

and observe that

$$
\begin{aligned}
\mathcal{U}^{\wedge}(m) & =\sum_{j=1}^{N} i m_{j} u_{j}^{Y}(m)|m|^{-2} & & \text { for } m \neq 0, \\
& =0 & & \text { for } m=0 .
\end{aligned}
$$

From (3.6) and (3.16), it follows that

$$
\int_{B\left(0, r_{1}\right)} \mathcal{U}(x) \Delta \phi(x) d x=0
$$

for all $\phi$ in $C_{0}^{\infty}\left[r_{1}, T_{N}\right]$, and we conclude once again from Weyl's lemma that

$$
\mathcal{U}(x)=\mathcal{U}^{\prime}(x) \text { almost everywhere in } B\left(0, r_{1}\right)
$$

$$
\text { where } \mathcal{U}^{\prime}(x) \text { is harmonic in } B\left(0, r_{1}\right) \text {. }
$$


From (3.17) and [6, Lemma 2], it follows that

$$
\lim _{t \rightarrow 0} \sum_{m \neq 0}|m|^{2} \mathcal{U}^{\wedge}(m) e^{i(m, x)-|m| t}=0 \text { for } x \text { in } B\left(0, r_{1}\right) \text {. }
$$

Also from (3.14) and [6, Lemma 2], it follows that

$$
\lim _{t \rightarrow 0} \sum_{m \neq 0}\left(\sum_{j=1}^{N} i m_{j} w_{j}^{\mathcal{Y}}(m)\right) e^{i(m, x)-|m| t}=\sum_{j=1}^{N} \frac{\partial w_{j}(x)}{\partial x_{j}} \text { for } x \text { in } B\left(0, r_{1}\right) .
$$

But from (3.14) and (3.16), we have

$$
\sum_{j=1}^{N} i m_{j} w_{j}^{\Upsilon}(m)-\nu|m|^{2} \mathcal{U}^{\wedge}(m)=\sum_{j=1}^{N} i m_{j} \nu_{j}^{\hat{j}(m)} \text { for } m \neq 0,
$$

and we conclude from (3.18), (3.19), and (3.20) that

(3.21) $\lim _{t \rightarrow 0} \sum_{m \neq 0}\left(\sum_{j=1}^{N} i m_{j} v_{j}^{\Upsilon}(m)\right) e^{i(m, x)-|m| t}=\sum_{j=1}^{N} \frac{\partial w_{j}(x)}{\partial x_{j}}$ for $x$ in $B\left(0, r_{1}\right)$.

Next, we set

$$
F_{j}(x)=(2 \pi)^{-N} \int_{T_{N}} f_{j}(y) H_{j}(x-y) d y
$$

and note from the hypothesis of the lemma and from Lemma 1 that

$$
F_{j}(x) \text { is a continuous periodic function }
$$

which in $C^{1+a}\left[B\left(0, r_{1}\right)\right]$ for $j=1, \cdots, N$.

Also we note from (3.22) that

$$
\begin{aligned}
F_{j}^{\hat{j}}(m) & =i m_{j} \hat{f_{j}(m)|m|^{-2}} & & \text { for } m \neq 0, \\
& =0 & & \text { for } m=0 .
\end{aligned}
$$

Next, we observe from (3.11) and (3.24) that

$$
\begin{aligned}
\sum_{j=1}^{N} i m_{j} v_{j}^{\wedge}(m)= & -p^{\wedge}(m)-\sum_{j=1}^{N} \sum_{k=1}^{N} m_{j} m_{k}\left(u_{j} u_{k}\right)^{\Upsilon}(m)|m|^{-2} \\
& -\sum_{j=1}^{N} F_{j}^{\wedge}(m) \text { for } m \neq 0 .
\end{aligned}
$$

Also, we observe that $u_{j} u_{k}$ is in $L \beta / 2\left[B\left(0, r_{1}\right)\right]$, and conclude from Lemmas 4, 6 and $11(a)$ that there is a function 


$$
W_{j k}(x) \text { in } L^{\beta / 2}[B(0, r)] \text { for every } r \text { such that } 0<r<r_{1}
$$

with the property that for $j, k=1, \cdots, N$

$$
\begin{aligned}
\lim _{t \rightarrow 0} \sum_{m \neq 0} m_{j} m_{k}|m|^{-2}\left(u_{j} u_{k}\right)^{\wedge}(m) e^{i(m, x)-|m| t} \\
=W_{j k}(x) \quad \text { almost everywhere in } B\left(0, r_{1}\right) .
\end{aligned}
$$

Also from [5, The orem 2, pp. 55-56], we have that

$$
\begin{aligned}
& \lim _{t \rightarrow 0} \sum_{m \neq 0} p^{\Upsilon}(m) e^{i(m, x)-|m| t}=p(x)-p^{\wedge}(0) \\
& \text { almost everywhere in } B\left(0, r_{1}\right),
\end{aligned}
$$

and from [5, Theorem 2] in conjunction with (3.23) and (3.24) that

$$
\lim _{t \rightarrow 0} \sum_{m \neq 0} F_{j}^{\wedge}(m) e^{i(m, x)-|m| t}=F_{j}(x) \text { for } x \text { in } B\left(0, r_{1}\right)
$$

We consequently conclude from (3.21), (3.25), (3.27), and (3.29) that

$$
\begin{aligned}
-p(x)+p^{\Upsilon}(0) & -\sum_{j=1}^{N} \sum_{k=1}^{N} W_{j k}(x)-\sum_{j=1}^{N} F_{j}(x) \\
& =\sum_{j=1}^{N} \frac{\partial w_{j}(x)}{\partial x_{j}} \text { almost everywhere in } B\left(0, r_{1}\right) .
\end{aligned}
$$

We rewrite this last fact as follows:

$$
p(x)=p^{\Upsilon}(0)-\sum_{j=1}^{N} \sum_{k=1}^{N} W_{j k}(x)-\sum_{j=1}^{N} F_{j}(x)
$$

$$
-\sum_{j=1}^{N} \frac{\partial w_{j}(x)}{\partial x_{j}} \text { almost everywhere in } B\left(0, r_{1}\right) .
$$

But then our desired result, namely (3.2), follows immediately from (3.14), (3.23), (3.26), and (3.30).

Next, we intend to show the following:

$$
\begin{aligned}
& \text { If } N<\beta<2 N, u_{j} \text { is in } L^{N \beta /(2 N-\beta)}[B(0, r)] \\
& \text { for every } r \text { such that } 0<r<r_{1}, j=1, \cdots, N \text {. }
\end{aligned}
$$

First, we observe from (3.2), (3.7), and Lemma 2 that 
(3.32) if $N<\beta<2 N, P_{j}$ is in $L^{N \beta /(2 N-\beta)}\left[B\left({ }^{\prime} 0, r\right)\right]$

for every $r$ such that $0<r<r_{1}$.

Likewise, since $u_{j} u_{k}$ is in $L^{\beta / 2}$ on compact subsets of $B\left(0, r_{1}\right)$, we have from Lemma 2 and (3.8) that

(3.33) if $N<\beta<2 N, U_{j k}$ is in $L^{N \beta /(2 N-\beta)}[B(0, r)]$

for every $r$ such that $0<r<r_{1}$.

Also, we have from the hypothesis of Lemma 12 and from (3.9) and Lemma 1 that

(3.34) $\mathcal{F}_{j}(x)$ is a continuous periodic function which

is in $C^{2+a}\left[B\left(0, r_{1}\right)\right]$.

Consequently, it follows from (3.32), (3.33), (3.34), and (3.10) that

(3.35) if $N<\beta<2 N, v_{j}$ is in $L^{N \beta /(2 N-\beta)}[B(0, r)]$ for

every $r$ such that $0<r<r_{1}$.

But then (3.31) follows immediately from (3.14) and (3.35).

Next, we observe the following:

(3.36) if $\beta=N+\epsilon$ where $0<\epsilon<N$, then $N \beta /(2 N-\beta) \geq N+2 \epsilon$.

As a consequence of (3.31) and (3.36) and working iteratively, we can assume that

(3.37) $u_{j}$ is in $L^{3 N / 2}[B(0, r)]$ for every $r$

such that $0<r<r_{1}, j=1, \cdots, N$.

But then $N(3 N / 2) /[2 N-3 N / 2]=3 N$ and we conclude from (3.31) and (3.37) that

(3.38) $u_{j}$ is in $L^{3 N}[B(0, r)]$ for every $r$ such that $0<r<r_{1}, j=1, \cdots, N$.

From (3.38), we obtain that

(3.39) $u_{j} u_{k}$ is in $L^{3 N / 2}[B(0, r)]$ for every $r$ such that $0<r<r_{1}$.

As a consequence we see from Lemmas 4, 6, and 11 (a) and (3.39) that the function $W_{j k}$ introduced in (3.26) and used in (3.27) actually is better than stated. 
In particular we now have that

$W_{j k}(x)$ is in $L^{3 N / 2}[B(0, r)]$ for every $r$

such that $0<r<r_{1}$

and that (3.27) still holds. Also (3.30) still holds, and we conclude from (3.30), (3.23), (3.14), and (3.40) that

(3.41) $p(x)$ is in $L^{3 N / 2}[B(0, r)]$ for every $r$

such that $0<r<r_{1}$.

But then it follows from (3.7), (3.41), and Lemma 3 that

(3.42) $\quad P_{j}$ is in $C^{1 / 6}[B(0, r)]$ for every $r$

such that $0<r<r_{1}$.

Likewise it follows from (3.8) and (3.39) that

(3.43) $U_{j k}$ is in $C^{1 / 6}[B(0, r)]$ for every $r$

such that $0<r<r_{1}$.

As a consequence, we obtain from (3.10), (3.34), (3.42) and (3.43) that there is a function $v_{j}^{\prime}$ with the following properties:

$$
\begin{aligned}
& v_{j}^{\prime} \text { is in } C^{1 / 6}[B(0, r)] \text { for every } r \\
& \text { such that } 0<r<r_{1} \text { and equals } v_{j} \text { almost } \\
& \text { everywhere in } T_{N^{*}} .
\end{aligned}
$$

But then from (3.14) and (3.44), we obtain that there is a function $u_{j}^{\prime}$ with the following properties:

(3.45) $u_{j}^{\prime}$ is in $C^{1 / 6}[B(0, r)]$ for every $r$

such that $0<r<r_{1}$ and equals $u_{j}$ almost

everywhere in $T_{N}$.

But then it follows from (3.45) and Lemmas 7, 8, and $11(\mathrm{~b})$ that the $W_{j k}$ in (3.26), (3.27), and (3.40) is much better than stated. In particular, we now have that

(3.46) $W_{j k}$ is in $C^{1 / 6}[B(0, r)]$ for every $r$ such that $0<r<r_{1}$.

Furthermore, we observe that (3.27) still holds and also that (3.30) still holds. We consequently conclude from (3.30), (3.23), (3.14), and (3.46) that there is a 
function $p^{\prime}(x)$ with the following properties:

(3.47) $p^{\prime}(x)$ is in $C^{1 / 6}[B(0, r)]$ for every $r$

such that $0<r<r_{1}$ and equals $p(x)$

almost everywhere in $T_{N^{*}}$.

But then it follows from (3.7), (3.47), and Lemma 1 that

(3.48) $P_{j}$ is in $C^{1+1 / 6}[B(0, r)]$ for every $r$

such that $0<r<r_{1}$.

Likewise it follows from (3.8) and (3.45) that

(3.49) $U_{j k}$ is in $C^{1+1 / 6}[B(0, r)]$ for every $r$

such that $0<r<r_{1}$.

As a consequence, we obtain from (3.10), (3.34), (3.48), and (3.49) that $v_{j}^{\prime}$ in (3.46) is such that

(3.50) $v_{j}^{\prime}$ is in $C^{1+1 / 6}[B(0, r]$ for every $r$

such that $0<r<r_{1}$.

But then from (3.14) and (3.50) we have that $u_{j}^{\prime}$ in (3.45) is such that

(3.51) $u_{j}^{\prime}$ is in $C^{1+1 / 6}[B(0, r)]$ for every $r$

such that $0<r<r_{1}$.

Next, it follows from (3.8), (3.51), and Lemma 1 that

(3.52) $U_{j k}$ is in $C^{2+1 / 6}[B(0, r)]$ for every $r$

such that $0<r<r_{1}$.

Also, we note from (3.8) that

$$
\begin{aligned}
\hat{U_{j k}^{\hat{k}}(m)} & =\left(u_{j} u_{k}\right)^{\wedge}(m) i m_{k}|m|^{\star 2} & & \text { for } m \neq 0, \\
& =0 & & \text { for } m=0 .
\end{aligned}
$$

As a consequence, for $t>0$,

$$
\frac{\partial U_{j k}(x, t)}{\partial x_{j}}=-\sum_{m \neq 0} m_{j} m_{k}|m|^{-2}\left(u_{j} u_{k}\right) \hat{)}(m) e^{i(m, x)-|m| t} .
$$

We conclude from $(3.52)_{z}(3.53)$, and $[6$, Lemma 2$]$ that the function $W_{j k}(x)$ introduced in (3.26) and used in (3.27) is such that 
(3.54)

$W_{j k}(x)$ is in $C^{1+1 / 6}[B(0, r)]$ for every $r$

such that $0<r<r_{1}$

and furthermore

$$
\lim _{t \rightarrow 0} \frac{\partial U_{j k}(x, t)}{\partial x_{j}}=W_{j k}(x) \text { everywhere in } B\left(0, r_{1}\right) .
$$

Since (3.30) still holds, and we conclude from (3.54), (3.14), and (3.23) that $p^{\prime}(x)$ in (3.47) is such that

(3.56) $p^{\prime}(x)$ is in $C^{1+\zeta}[B(0, r)]$ for every $r$

such that $0<r<r_{1}$ where $\zeta=\min [\alpha, 1 / 6]$.

But then it follows from (3.7), (3.56), and Lemma 1 that

(3.57) $P_{j}$ is in $C^{2+\zeta}[B(0, r)]$ for every $r$ such that $0<r<r_{1}$.

As a consequence, we obtain from (3.10), (3.44), (3.34), (3.52), and (3.57) that

(3.58) $v_{j}^{\prime}$ is in $C^{2+\zeta}[B(0, r)]$ for every $r$ such that $0<r<r_{1}$.

From (3.14), (3.45) and (3.58), we obtain $u_{j}^{\prime}$ is in $C^{2+\zeta}[B(0, r)]$ for every $r$ such that $0<r<r_{1}$.

But then it follows from (3.8), (3.59), and Lemma 1 that

(3.60) $U_{j k}$ is in $C^{3+\zeta}[B(0, r)]$ for every $r$ such that $0<r<r_{1}$.

As a consequence, we see that we can replace (3.54) with the statement

(3.61) $W_{j k}(x)$ is in $C^{2+\zeta}[B(0, r)]$ for every $r$ such that $0<r<r_{1}$.

But (3.30) still holds, and we conclude from (3.14), (3.23), and (3.61) that

(3.62) $p^{\prime}(x)$ is in $C^{1+a}[B(0, r)]$ for every $r$ such that $0<r<r_{1}$.

Next, it follows from (3.7), (3.62), and Lemma 1 that

(3.63) $P_{j}$ is in $C^{2+a}[B(0, r)]$ for every $r$ such that $0<r<r_{1}$. 
As a consequence, we obtain from (3.10), (3.34), (3.60), and (3.63) that

(3.64) $v_{j}^{\prime}$ is in $C^{2+a}[B(0, r)]$ for every $r$

such that $0<r<r_{1}$.

But then from (3.14) and (3.64), we obtain that

(3.65) $u_{j}^{\prime}$ is in $C^{2+a}[B(0, r)]$ for every $r$

such that $0<r<r_{1}$.

The conclusion to the lemma follows immediately from (3.45), (3.47), (3.62), and (3.65), and the proof of the lemma and consequently the theorem is complete.

4. Proof of Theorem 2. To prove Theorem 2 we can assume, with no loss in generality, that

$$
0<r_{1}<r_{0}<1
$$

and that

$$
u, p \text {, and } f=0 \text { in } T_{N}-B\left(0, r_{0}\right)
$$

Also, we assume that

$$
(u, p) \text { is a distribution solution of }(1.1) \text { in } B\left(0, r_{1}\right)-\{0\}
$$

and observe from (4.1), (4.2), and (4.3) that the theorem will be established once we establish the following fact:

$$
\text { For } \phi \text { in } C_{0}^{\infty}\left[r_{1}, T_{N}\right] \text {, both (3.5) and (3.6) are valid, }
$$

where with no loss in generality we have assumed that (3.1) holds.

In order to establish (4.4), we say

(4.5) $\phi$ is in $C_{0}^{\infty}\left[r_{1}, T_{N}-\{0\}\right]$ if $\phi$ is in $C_{0}^{\infty}\left[B\left(0, r_{1}\right)-\{0\}\right]$ and

$$
\phi=0 \text { in } T_{N}-B\left(0, r_{1}\right) \text {. }
$$

We observe from (4.3) that the following fact does hold:

$$
\text { For } \phi \text { in } C_{0}^{\infty}\left[r_{1}, T_{N}-\{0\}\right] \text {, both (3.5) and (3.6) are valid. }
$$

Next, we let $P_{j}, U_{j k}, \mathcal{F}_{j k}$, and $v_{j}$ be defined almost everywhere in $T_{N}$ by 
(3.7), (3.8), (3.9), and (3.10) respectively and observe from (3.11) and (4.6) that

$$
\text { For } \phi \text { in } C_{0}^{\infty}\left[r_{1}, T_{N}-\{0\}\right],(3.12) \text { holds. }
$$

From (4.7), we conclude that (3.13) holds for all $\phi$ in $C_{0}^{\infty}\left[r_{1}, T_{N}-\{0\}\right]$. As a consequence we conclude from Weyl's lemma that for $j=1, \cdots, N$ there is a function $w_{j}(x)$ such that

$$
\begin{aligned}
& w_{j}(x) \text { is harmonic in } B\left(0, r_{1}\right)-\{0\} \text { and } \\
& \text { equal almost everywhere in } T_{N} \text { to } \nu u_{j}(x)+v_{j}(x) \text {. }
\end{aligned}
$$

From condition (ii) in the hypothesis of the theorem and Schwarz's inequality, we have for $j, k=1, \cdots, N$ that

$$
r^{-N} \int_{B(0, r)}\left|u_{j} u_{k}\right| d x=o\left(r^{-(N-1)}\right) \text { as } r \rightarrow 0 \text { for } N \geq 3
$$

[We note for future reference that (4.9) is also true for $N=2$.]

As a consequence, we conclude from condition (i) in the hypothesis of the theorem, from (4.9), (3.7), and (3.8), and from [7, Lemma 4] that for $j, k=1, \cdots$, $N$ both

$$
\begin{aligned}
& r^{-N} \int_{B(0, r)}\left|P_{j}\right| d x=o\left(r^{-(N-2)}\right), \text { as } r \rightarrow 0 \text { and } \\
& r^{-N} \int_{B(0, r)}\left|U_{j k}\right| d x=o\left(r^{-(N-2)}\right), \text { as } r \rightarrow 0 \text { for } N \geq 3 .
\end{aligned}
$$

Likewise, we obtain from (3.9) and Lemma 13 that

$$
r^{-N} \int_{B(0, r)}\left|\mathcal{F}_{j}\right| d x=0\left(r^{-(N-2)}\right) \text { as } r \rightarrow 0 \text { for } N \geq 3 \text {. }
$$

We consequently conclude from condition (ii) in the hypothesis of the theorem, from (4.10), (4.11) and (3.10) and from (4.8) that for $j=1, \cdots, N$

$$
r^{-N} \int_{B(0, r)}\left|w_{j}\right| d x=o\left(r^{-(N-2)}\right) \text { as } r \rightarrow 0 \text { for } N \geq 3 \text {. }
$$

Using the theory of spherical harmonics as in [7, (3.61) and (3.62), p. 94], we conclude from (4.8) and (4.12) that the following holds:

(4.13) For $N \geq 3, w_{j}$ can be defined at 0 so that $w_{j}$ is harmonic in $B\left(0, r_{1}\right)$.

Next, for $N \geq 2$, we define $U(x)$ almost everywhere in $T_{N}$ by (3.15) and observe from (4.6), (3.6), and (3.16) that 


$$
\int_{B\left(0, r_{1}\right)} \mathcal{U}(x) \Delta \phi(x) d x=0 \text { for } \phi \text { in } C_{0}^{\infty}\left[r_{1}, T_{N}-\{0\}\right] .
$$

From (4.14) and Weyl's lemma, we conclude that

(4.15) $\mathcal{U}(x)=\mathcal{U}^{\prime}(x)$ almost everywhere in $B\left(0, r_{1}\right)$

where $U^{\prime}(x)$ is harmonic in $B\left(0_{2} r_{1}\right)-\{0\}$.

From condition (ii) in the hypothesis of the theorem, we obtain

$$
r^{-N} \int_{B(0, r)}\left|u_{j}\right| d x=o\left(r^{-(N-1) / 2}\right) \text { as } r \rightarrow 0
$$

for $N \geq 2$ and $j=1, \cdots, N$.

But then we obtain from [7, Lemma 4] and (3.15) that

$$
r^{-N} \int_{B(0, r)}|\mathrm{U}| d x=o\left(r^{-(N-2)}\right) \text { for } N \geq 3
$$

$$
=o\left(\log r^{-1}\right) \text { for } N=2 \text {, as } r \rightarrow 0 .
$$

We conclude from the theory of spherical harmonics that

(4.18) From $N \geq 2, \mathrm{U}^{\prime}$ can be defined at the origin so that it is harmonic in $B\left(0, r_{1}\right)$.

Next, we show that (4.13) holds also for $N=2$. To see this we observe from [7, Lemma 4] and from Lemma 13 that

(4.19) For $N=2$, the expressions on the right-hand side of the equality sign in (4.10) and (4.11) are to be replaced by $o\left(\log r^{-1}\right)$.

From (4.8), (3.10), (4.16), and (4.19), we next obtain that

$$
r^{-2} \int_{B(0, r)}\left|w_{j}\right| d x=0\left(r^{-1 / 2}\right) \text { as } r \rightarrow 0 \text { for } j=1,2
$$

Using the theory of spherical harmonics as before we conclude from (4.8) and (4.20) that for $j=1,2$,

(4.21) $w_{j}(x)=c_{j} \log |x|+G_{j}(x)$ for $x$ in $B\left(0, r_{1}\right)-\{0\}$ where $c_{j}$ is a constant and $G_{j}(x)$ is harmonic in $B\left(0, r_{1}\right)$.

From (4.15) and (4.21), we have that (3.18) and (3.19) hold for $x$ in $B\left(0, r_{1}\right)-$ \{0\}. We therefore obtain from (3.20) that (3.21) holds for $x$ in $B\left(0, r_{1}\right)-\{0\}$. We write this out as follows: 


$$
\begin{array}{r}
\sum_{j=1}^{N} c_{j} x_{j}|x|^{-2}+\sum_{j=1}^{N} \frac{\partial G_{j}(x)}{\partial x_{j}}=\lim _{t \rightarrow 0} \sum_{m \neq 0}\left(\sum_{j=1}^{N} i m_{j} v_{j}^{\hat{\gamma}(m)}\right) e^{i(m, x)-|m| t} \\
\text { for } x \text { in } B\left(0, r_{1}\right)-\{0\} .
\end{array}
$$

Next, we define $F_{j}(x)$ almost everywhere in $T_{N}$ by (3.22) and observe that (3.24) and (3.25) hold. Also from condition (ii) in the hypothesis of the theorem, we have that

(4.23) there is an $r_{2}$ with $0<r_{2}<r_{1}$ such that $u_{j} u_{k}$

is in $L^{\gamma / 2}\left[B\left(0, r_{2}\right)\right]$ for $j, k=1,2$.

Since $\gamma>2$, we conclude from (4.23), (3.25), Lemmas 6 and 11 that

(4.24) there is an $r_{3}$ with $0<r_{3}<r_{2}$ and a

function $M(x)$ in $L^{1}\left[B\left(0, r_{3}\right)\right]$ such that

the expression on the right-hand side of the equality sign in (4.22) is equal to $M(x)$

almost everywhere in $B\left(0, r_{3}\right)$.

From (3.22) and Lemma 13, we have that

$$
r^{-2} \int_{B(0, r)} \sum_{j=1}^{N}\left|F_{j}\right| d x=o\left(r^{-1}\right) .
$$

From condition (ii) in the hypothesis of the theorem, we have that

$$
\left\{r^{-2} \int_{B(0, r)}\left|u_{j} u_{k}\right|^{\gamma / 2} d x\right\}^{2 / \gamma}=o\left(r^{-1}\right)
$$

where with no loss in generality we can also assume that

$$
2<\gamma<4 \text {. }
$$

Now it follows from (3.25) and (4.24) that

$$
M(x)+p(x)-p^{\wedge}(0)+\sum_{j=1}^{N} F_{j}(x)
$$

$$
=-\lim _{t \rightarrow 0} \sum_{m \neq 0}\left[\sum_{j=1}^{N} \sum_{k=1}^{N} m_{j} m_{k}\left(u_{j} u_{k}\right)^{\wedge}(m)\right] e^{i(m, x)-|m| t}
$$

almost everywhere in $B\left(0, r_{3}\right)$.

We conclude from condition (i) in the hypothes is of the theorem, from (4.25), (4.23), (4.26), (4.27), Lemma 12, and (4.28) that 


$$
r^{-2} \int_{B(0, r)}|M(x)| d x=o\left(r^{-1}\right) \text { as } r \rightarrow 0 .
$$

From (4.21), we have that $G_{j}(x)$ is harmonic in $B\left(0, r_{1}\right)$ for $j=1, \cdots, N$. Therefore $\partial G_{j}(x) / \partial x_{j}$ is harmonic in $B\left(0, r_{1}\right)$. Since $r_{3}<r_{1}$, we conclude from (4.22) and (4.24) that

(4.30) there is a function $G(x)$ harmonic in $B\left(0, r_{3}\right)$ such that

$$
\sum_{j=1}^{2} c_{j} x_{j}|x|^{-2}=M(x)+G(x) \text { almost everywhere in } B\left(0, r_{3}\right) \text {. }
$$

From (4.30) we obta in that

$$
\begin{aligned}
c_{k} \int_{B(0, r)} x_{k}^{2}|x|^{-3} d x=\int_{B(0, r)}(M+G) x_{k}|x|^{-1} d x \\
\text { for } 0<r<r_{3} \text { and } k=1,2 .
\end{aligned}
$$

From (4.29), (4.30), and (4.31) we further obtain that

$$
c_{k} \pi r=o(r) \quad \text { for } k=1,2 .
$$

Therefore $c_{k}=0$ for $k=1,2$, and we conclude from (4.21) that

(4.33) for $N=2, w_{j}$ can be defined at 0 so that it is harmonic in $B\left(0, r_{1}\right)$.

From (4.13) and (4.33), we obtain that

$$
\int_{T_{N}} w_{j}(x) \Delta \phi(x) d x=0 \text { for } \phi \text { in } C_{0}^{\infty}\left[r_{1}, T_{N}\right] \text { and } j=1, \ldots, N .
$$

Likewise from (4.15) and (4.18), we have

$$
\int_{T_{N}} U(x) \Delta \phi(x) d x=0 \text { for } \phi \text { in } C_{0}^{\infty}\left[r_{1}, T_{N}\right]
$$

From (4.8), we have that $w_{j}(x)=\nu u_{j}(x)+v_{j}(x)$ almost everywhere in $T_{N^{*}}$ From this fact, (4.34), and (3.11) we see that (3.5) holds for $\phi$ in $C_{0}^{\infty}\left[r_{1}, T_{N}\right]$. Likew ise from (3.16) and (4.35), we see that (3.6) holds for $\phi$ in $C_{0}^{\infty}\left[r_{1}, T_{N}\right]$. Consequently (4.4) is established and the proof of Theorem 2 is complete.

\section{REFERENCES}

1. A. P. Calderón and A. Zygmund, Singular integrals and periodic functions, Studia Math. 14 (1954), 249-271. MR 16, 1017.

2. Günter Hellwig, Partielle Differentialgleichungen: Eine Einführung, Mathematische Leitfäden, Teubner Verlagsgesellschaft, Stuttgart, 1960; English transl., Blaisdell, Waltham, Mass., 1964. MR 22 \#5794; MR 30 \#3286. 
3. O. A. Ladyženskaja, Mathematical problems in the dynamics of a viscous incompressible flow, Fizmatgiz, Moscow, 1961; English rev. ed., Gordon and Breach, New York, 1969. MR 27 \#5034a; MR 40 \#7610.

4. V. L. Shapiro, Topics in Fourier and geometric analysis, Mem. Amer. Math. Soc. No. 39 (1961). MR 26 \#5339.

5. - Fourier series in several variables, Bull. Amer. Math. Soc. 70 (1964), 4893. MR $28 \# 1448$.

6. - Characteristic planes and pointwise solutions of the heat equation, Indiana Univ. Math. J. 20 (1971), 115-133. MR 41 \#8844.

7. - Removable sets for pointwise solutions of the generalized Cauchy-Riemann equations, Ann. of Math. (2) 92 (1972), 82-101.

8. L. Bers, F. John and M. Schechter, Partial differential equations, Lectures in Appl. Math., vol. 3, Interscience, New York, 1964. MR 29 \#346.

9. A. P. Calderón and A. Zygmund, Local properties of solutions of elliptic partial differential equations, Studia Math. 20 (1961), 171-225. MR 25 \#310.

10. R. H. Dyer and D. E. Edmunds, Removable singularities of solutions of the Navier-Stokes equations, J. London Math. Soc. (2) 2 (1970), 535-538. MR 42 \#697.

DEPARTMENT OF MATHEMATICS, UNIVERSITY OF CALIFORNIA, RIVERSIDE, CALIFORNIA 92502 